\section{Uterine Leiomyoma or GIST? A Case Report of a Diagnostic Conundrum}

\section{Abstract}

Uterine leiomyomas are the most common tumours to affect women. The presentation of these tumours can vary. Typical uterine leiomyomas are easily recognized on imaging. However, an atypical presentation or even atypical appearances on imaging can cause diagnostic confusion. We report a case of uterine leiomyoma presenting itself as a gastrointestinal tumour (GIST) on imaging. She underwent a laparotomy and was found to have a uterine leiomyoma necessitating resection. She was subsequently discharged well.

\section{See A, Tan G and Teo M}

Division of Surgical Oncology, National Cancer Centre, Singapore

\section{Corresponding author: Dr Amanda See}

\section{amanda.see@mohh.com.sg}

MBBS, MRCS, Division of Surgical Oncology, National Cancer Centre, Singapore

Tel: $+(65) 64368283$;

Fax: (65) 62257559 ;

\section{Introduction}

Uterine leiomyomas are the most common tumours to affect women, and can cause pelvic discomfort and menorrhagia [1]. On the other hand, gastrointestinal stromal tumours (GISTs) are the most common mesenchymal tumours of the gastro-intestinal tract, and are often asymptomatic [2]. There have been few reports of uterine fibroids mimicking other tumors, a situation where the diagnosis is challenging and may occasionally result in a misdiagnosis. Here we report a case, and present the impressive images for a case in which a lady with uterine leiomyoma was initially thought to have a colonic GIST.

\section{Case Report}

Madam S is a 41 year old Indonesian lady who has a past medical history of uterine fibroids, on follow-up with a gynaecologist. She presented to the hospital with complaints of severe right upper quadrant pain of 2 days duration. There was no fever, change in bowel habits, per-rectal bleeding or vomiting. On examination, she was alert but in mild distress. She was not pale, nor jaundiced. Her abdomen was tender over the right upper quadrant with localized guarding but no evidence of peritonism. There was also a large mass felt over the same region. Hematological investigations were largely unremarkable except for a hemoglobin of $10 \mathrm{~g} / \mathrm{dl}$ (normal range: $12-15.5 \mathrm{~g} / \mathrm{dl}$ ) and raised total white cell count of $14.5^{\wedge} 1000$ (normal range: 4.50 to $10.0^{\wedge} 1000$ ). Tumour markers were performed, and the CA-125 was noted to be raised at 71.4 $\mathrm{U} / \mathrm{ml}$ (normal range: $<35 \mathrm{U} / \mathrm{ml}$ ).

A CT scan of her abdomen and pelvis was done urgently on the same day of admission. Findings on the scan showed a large heterogenous mass in the upper abdomen, displacing the adjacent structures and was inseparable from the proximal transverse colon (Figures 1-3). A discussion was held with the radiologists who felt that the differential diagnoses would include a colonic gastrointestinal stromal tumour (GIST) and mesenchymal tumour of the mesentery. In addition, other findings include a possible fibroid in the posterior uterine wall and bilateral cystic masses (Figure 4). Her case was discussed at the multi-disciplinary meeting which concurred with the above findings.

She was counselled for an exploratory laparotomy and resection of the large mass, enbloc with colonic resesction, and myomectomy for her fibroids. In addition she was also advised that a total abdominal hysterectomy and bilateral salphingo-oopherecetomy may be necessary if there was excessive bleeding during the surgery. She was agreeable and underwent surgery.

Intra-operative findings did not reveal any GIST. Instead, there was a 16 weeks sized uterus with a large $15 \mathrm{~cm}$ pedunculated fibroid at the fundus, extending up the right hypochondrium. This large fibroid had been mistaken for the colonic GIST on the $\mathrm{CT}$ abdomen and pelvis (Figure 5). The transverse colon was not found to be adherent to the mass. An on-table consult with the gynaecologists was made, and she underwent resection of the pedunculated fibroid as well as myomectomies of her other uterine fibroids.

Her post-operative recovery was uneventful and she was discharged well on post-operative day 2 . The final histology was uterine leiomyoma.

\section{Discussion}

Uterine leiomyomas are the most common benign gynaecological tumours. They appear as round, well circumscribed solid nodules and exhibit a classically described 'whorled-like' apperance on histological analysis. A typical leiomyoma is easily diagnosed on imaging, and most of them may be confidently diagnosed using sonography. The presentation of uterine leiomyomas can differ 


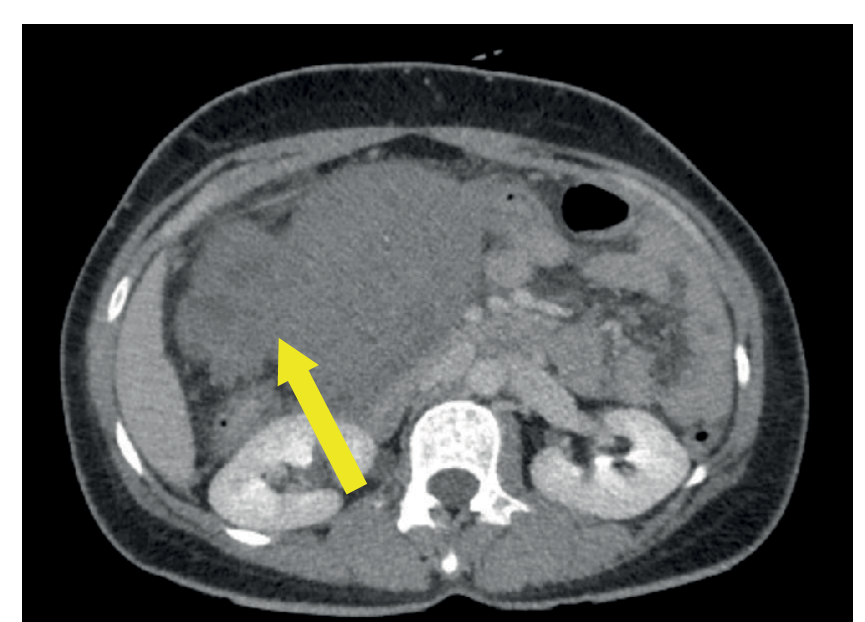

Figure 1 Arrow showing mass that is inseparable from the transverse colon.

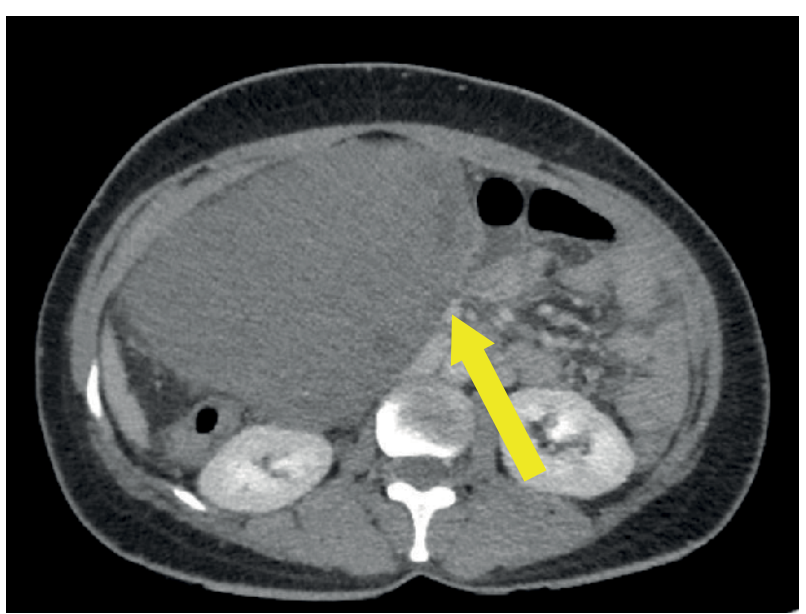

Figure 2 Arrow showing large heterogenous mass in the right upper abdomen.

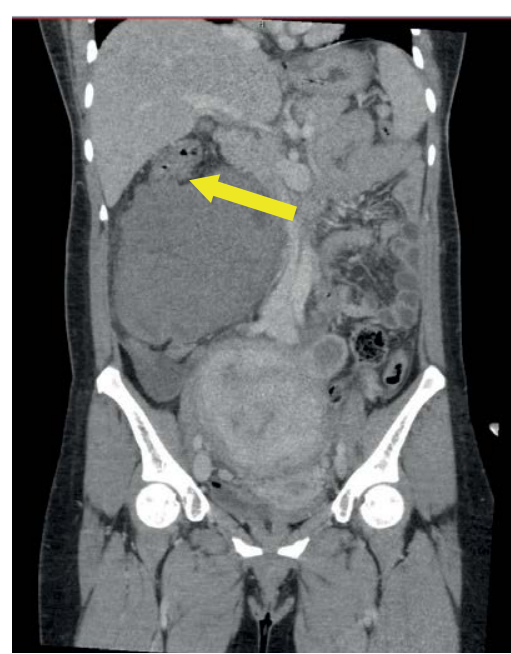

Figure 3 CT scan showing a the mass that is inseparable from the colon, in coronal section.

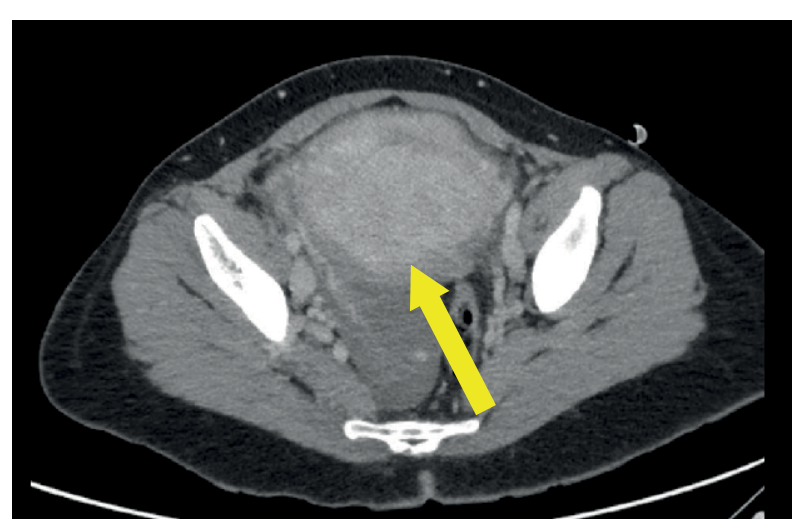

Figure 4 Arrow showing uterine wall mass, likely a uterine fibroid.

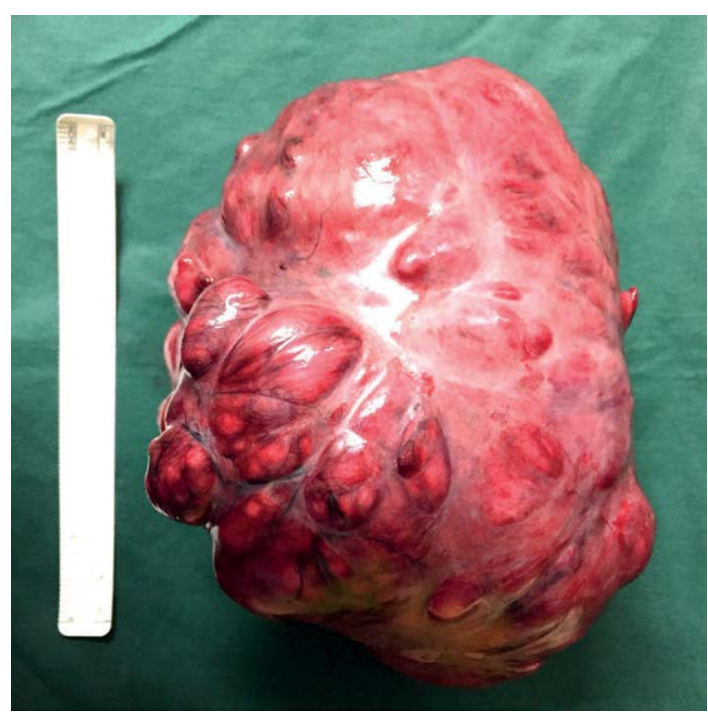

Figure 5 Mass that was resected measuring $15 \mathrm{~cm}$ - final histology came back as uterine leiomyoma.

from patient to patient [1]. They do give rise to complications such as bleeding, anemia, defective implantation of an embryo and pelvic discomfort [3]. In addition, if large, they may mimick other malignant condition, with a case report describing a retroperitoneal leiomyoma mimicking a sarcoma [4]. There also have been other case reports which describes uterine leiomyoma mimicking GIST [5] as well as mimicking an abdominal mass [6] which presented as a diagnostic conundrum to the clinicians. This definitely presents as a diagnostic challenge as management of the patient would then differ.

GISTs constitue the majority of gastrointestinal mesenchymal tumours of the GIT and are known to be refractory to conventional chemotherapy or radiation. They form $0.1 \%-3 \%$ of gastrointestinal malignant tumours $[7,8]$. Up to $70 \%$ of patients with GIST are symptomatic, with vague abdominal discomfort being the most common complaint (60-70\%). The imaging of choice is a contrasted computed tomography (CT) scan and they appear as large, well-defined oft tissue mass with heterogenous enhancement. They are usually of varying density and show patchy enhancement after intravenous contrast. 
In our present case report, our patient had in fact a uterine leiomyoma but in fact was thought to be a GIST initially. This led to a misdiagnosis. There has so far been only one reported case of uterine leiomyoma mimicking a GIST and this was only discovered on final histology analysis [5]. As mentioned, sonographic imaging methods are the primary and perhaps most cost effective modalities for the detection of leiomyomas [5]. Leiomyomas can undergo degenerative changes and this can alter the apperance on computed tomography scan, plus the fact that our patient had a large mass. The mass also appeared to be arising from the mesentry and seemed to be contiguous with the transverse colon, leading to the impression that the mass was a GIST. Furthermore, we did not have a histological diagnosis prior to the laparotomy, as her persistent abdominal pain nesssitated an emergency procedure.

Diagnosing uterine leiomyomas can be difficult and challenging if the appearance of the uterine mass is not typical on imaging. They classically appear solid in echogenic terms (though may be slightly hypoechoic if through-transmission or penetration is poor) [9]. However, if the uterine leiomyoma is pedunculated, the origin of the pedunculated leiomyoma may be obscure and perhaps a CT or even MRI imaging may assist in diagnosis. Perhaps in our case, we could have proceeded with an MRI, but it was thought that the mass was almost certainly a GIST. This may be because the leiomyoma have adhered to the bowels or mesentry.

As the presentation of uterine leiomyomas can vary, there have been a few case reports reporting uterine leiomyomas presenting themselves as GIST, retroperitoneal sarcoma [4] or even abdominal masses [6]. Zhu et al. [5] stated in his paper the difficulties in diagnosing the pelvic mass which was initially thought to be a GIST. Even after resection, the pathological report indicated a diagnosis of spindle cell tumour and multiple stains had to be performed before the diagnosis of leiomyoma could be ascertained. He suggested that perhaps a biopsy could be performed prior to resection. However, if the patient presents acutely with abdominal pain necessitating a laparotomy, this approach might be not be feasible.

This highlights the importance of clinching the diagnosis as the management and prognosis would differ if it was a malignant condition instead of a uterine leiomyoma. Jeong G.U. [4] has reported a case of retroperitoneal leiomyoma mimicking a sarcoma in which the mass was still thought to be a sarcoma even on MRI imaging. The patient was then planned for a radical resection. They found a tumour which was attached to the uterus and histology came back as subserosal uterine leiomyoma. The prognosis for a sarcoma varies vastly from that of a uterine leiomyoma - it is poor because of aggressive course and high local recurrence rates [10].

There have been no further reports of uterine leiomyomas mimicking GIST and hence, no guidelines exist concerning appropriate recommended procedures for differential diagnosis. This case report aims to highlight the fact that even though rare, uterine leiomyomas can mimic the apperance of a GIST. It can affect the operative plan and further adjuvant therapy postoperatively. The diagnosis of a uterine leiomyoma should be considered if the apperance of a GIST on CT scan is in debate. However, if unable to exclude a GIST, exploratory laparatomy should be performed. 


\section{References}

1 Gupta S (2009) Acute complications of fibroids. Best Prac Res Clin Obstetrics Gynaecology 23: 609-617.

2 Miettinen M, Lasota J (2001) Gastrointestinal stroma tumours definition, clinical histological, immunohistochemical and molecular genetic features and differential diagnosis. Virchows Arch 438: 1-12.

3 Bulun SE (2013) Uterine fibroids. N Engl J Med 369: 1344-1355.

4 Jeong GA (2014) Retroperitoneal leiomyomas of the uterus mimicking sarcoma in perimenopausal women: Case report. J Menopausal Med 20:133-137.

5 Kim KW (2005) Incidence of GIST in Koreans: It's incidence and the clinical, pathologic and immunohistochemical findings. J Korean med Sci 20: 977-984.
6 Goettsch WG (2005) Incidence of GIST is underestimated: results of a nation-wide study. Eur J Cancer 41: 2868-2872.

7 Zhu XQ (2015) Uterine leiomyoma mimicking a gastrointestinal stromal tumour with chronic spontaneous hemorrhadge: A case report. Oncology letters s9: 2481-2484.

8 Low SC, Chong CL (2004) A case of cystic leiomyoma mimicking an ovarian malignancy. Ann Acad med Singapore 33: 371-374

9 Tugee O (2015) A giant subserosal uterine leiomyoma mimicking an abdominal mass: Multimodal imaging data. Acta Medica Iranica 53: 4.

10 Erzen D, Sencar M, Novak J (2005) Retroperitoneal sarcoma: 25 years of experience with aggressive surgical treatment at the Institute of Oncology, Ljubljana. J Surg Oncol 91: 1-9. 